Soziale Demokratie? Die politökonomische Heterogenität Europas als Determinante des demokratischen und sozialen Potenzials der EU

EuR-Bei 2013 69

\title{
Soziale Demokratie? Die politökonomische Heterogenität Europas als Determinante des demokratischen und sozialen Potenzials der EU \\ Von Martin Höpner, Köln ${ }^{*}$ \\ I. Einleitung: Soziale Demokratie in Europa ${ }^{1}$ \\ Martin Höpner: Soziale Demokratie? Die politökonomische Heterogenität Europas als Determinante des demokratischen und sozialen Potenzials der EU. In: Europarecht Beiheft 48(1), 69-89 (2013). Nomos

Dem Begriff der Sozialen Demokratie wohnt eine theoretisch ebenso wie praktisch weitreichende Doppelbedeutung inne. Erstens, die Gestaltungskraft der Politik darf vor der Ökonomie nicht Halt machen, sondern muss sich auf die wirtschaftlichen und sozialen Lebensverhältnisse der Bürgerinnen und Bürger erstrecken. Zweitens, die sozialen Verhältnisse entfalten ihrerseits Rückwirkungen auf die Demokratie. Bleibt Teilen der Gesellschaft der Zugang zu wirtschaftlichen Ressourcen, Kultur und Bildung verwehrt, so die Verfechter der Sozialen Demokratie, dann fehlt der Demokratie eine entscheidende Funktionsvoraussetzung. Sie läuft Gefahr, "nur" politische Demokratie zu bleiben, eine leere, formale Hülle, die sich nicht mit Leben füllen kann. ${ }^{2}$

Aus diesem Doppelsinn folgt ein zweifacher Auftrag an das Recht und an die Politik. Formale demokratische Strukturen sind auszubauen und zu pflegen, diesem Teil des Auftrags würden auch liberale Denker nicht widersprechen. Aber der demokratiebezogene Teil des Auftrags reicht weiter, denn die Soziale Demokratie lenkt die Aufmerksamkeit auf das Verhältnis zwischen den Subjekten und Objekten politischer Steuerung. ${ }^{3}$ Soziale Demokratie setzt die politische Steuerbarkeit des Wirtschaftlichen und des Sozialen voraus sowie eine entsprechende Steuerungsfähigkeit demokratischer Politik. Anders ausgedrückt: Das Primat der Politik ist Voraussetzung der Sozialen Demokratie. Würden Regierungen nach allen Regeln demokratischer Kunst gewählt und kontrolliert, ohne aber steuernde Eingriffe in Produktion und Distribution vornehmen zu können, wäre die Demokratie sozial entleert.

Gleichzeitig folgt aus der Lehre von der Sozialen Demokratie auch ein spezifischer inhaltlicher Auftrag. Fungiert das Soziale als Funktionsvoraussetzung der

Soziale Demokratie? Die politökonomische Heterogenität Europas als Determinante des demokratischen und sozialen Potenzials der EU (EuR-Bei 2013, 69)

Demokratie, dann diktiert der demokratische Auftrag gleichzeitig eine Vorauswahl zwischen konkurrierenden Politikoptionen. Die demokratische Inklusion kann dieser Sicht zufolge nur gelingen, wenn ihr die materielle Inklusion schwacher Bevölkerungsschichten an die Seite gestellt wird. Verfechter der Sozialen Demokratie favorisieren politische Eingriffe zur Verminderung sozialer Ungleichheit sowie zur Stärkung der Sozialbindung privaten Eigentums, ein progressives Steuersystem und die Bereitstellung subventionierter Infrastrukturdienstleistungen durch die öffentliche Hand. Diesen Idealen kommen die skandinavischen Länder vergleichsweise nahe, gefolgt von kontinentaleuropäischen Wohlfahrtsstaaten wie Deutschland, Frankreich und den Niederlanden. ${ }^{4}$

Prüft man nun die Europäische Union auf die Potenziale einer so verstandenen Sozialen Demokratie, wird man zu einem gemischten Ergebnis gelangen. Immerhin, die Grundlagen europäischer Sozialer Demokratie scheinen in Entwicklung begriffen, mühevoll und langsam zwar, aber stetig und in die erwünschte Richtung. Die mit dem Vertrag von Lissabon eingeleiteten Reformen der EU versprechen einen Zugewinn an Demokratie, denn das Europäische Parlament, die einzige direkt gewählte EU-Institution, erfährt eine Aufwertung. Das Mitentscheidungsverfahren wurde auf zahlreiche neue Politikfelder wie die Gemeinsame Agrarpolitik und die justizielle Zusammenarbeit in Strafsachen ausgedehnt und damit - so der neue Terminus - zu dem ordentlichen Gesetzgebungsverfahren ausgebaut (Art. 294 AEUV). Nunmehr existieren praktisch keine Aufgabenbereiche der EU mehr, über die ohne Beteiligung des EP beschlossen werden kann. Das neue parlamentarische Recht, die Kommission zu Gesetzesinitiativen aufzufordern, kann als Vorstufe zu einem parlamentarischen Initiativrecht gedeutet werden. Eine weitere gute Nachricht für Verfechter der Sozialen Demokratie ist die Europäische Bürgerinitiative: Mit Volksinitiativen, die von mindestens einer Million europäischer Bürgerinnen und Bürger aus mehreren Mitgliedsländern unterstützt werden, können die EU-Organe zum Handeln aufgefordert werden.

Gute Nachrichten im Sinne der Sozialen Demokratie gibt es auch in Bezug auf den an die EU-Organe gerichteten sozialen Auftrag. Dem Ziel der Verwirklichung eines gemeinsamen Binnenmarktes werden immer mehr soziale Ziele an die Seite gestellt. Bereits vor der Verabschiedung des Lissabon-Vertrags hat der EuGH soziale Grundrechte anerkannt, die Einschränkungen der wirtschaftlichen Grundfreiheiten zu rechtfertigen vermögen. Nunmehr wird die Charta der Grundrechte für das Handeln der europäischen Organe rechtsverbindlich, die auch soziale Grundrechte enthält, so das Recht auf Unterrichtung und Anhörung der Arbeitnehmer und das Recht auf Kollektivverhandlungen sowie auf Kollektivmaßnahmen (zu denen auch der Streik zählt). Das europäische Primärrecht enthält - in Art. $\underline{3}$ EUV - nunmehr ein Bekenntnis zur "soziale(n) Marktwirtschaft, die auf Vollbeschäftigung und sozialen Fortschritt abzielt". Die EU, so Art. 152 AEUV, anerkennt und fördert die Rolle der

Soziale Demokratie? Die politökonomische Heterogenität Europas als Determinante des demokratischen und sozialen Potenzials der EU (EuR-Bei 2013,69)

Sozialpartner und achtet ihre Autonomie. Zahlreiche weitere Verweise auf das Soziale im europäischen Primär- und Sekundärrecht ließen sich nennen. Aus guten Gründen, so sollte man meinen, konnte die deutsche Bundeskanzlerin aus Anlass der Ratifizierung des EU-Reformvertrags durch den Deutschen Bundestag auf den 60. Geburtstag der sozialen Marktwirtschaft deutschen Typs verweisen und feststellen: „Unsere Europäische Union ist den gleichen Werten verpflichtet, wie wir sie im deutschen Sozialmodell kennen." Und nichts, so ließe sich weiter argumentieren, deutet darauf hin, dass sich der soziale und demokratische Gehalt der EU nicht weiter steigern ließe. So erkennen soziale Bewegungen in der EU weiteres, derzeit noch ungenutztes soziales und demokratisches Potenzial und streben danach, es mit Leben zu füllen. Als Beispiel sei der in Sozialdemokratie und Gewerkschaften diskutierte "soziale 
Stabilitätspakt" genannt, der unter anderem auf eine Verpflichtung der Mitgliedstaaten zielt, ihre Sozialausgaben in bestimmten Bandbreiten zu halten und somit etwaigen Unterbietungswettläufen entgegenzuwirken. ${ }^{6}$ Kurz, die europäische Soziale Demokratie scheint in Entwicklung begriffen und lässt weitere Potenziale erkennen, die sich nutzen lassen, wenn es ihren Verfechten gelingt, politische Mehrheiten für ihre Vorstellungen zu mobilisieren.

Dieser optimistischen Interpretation möchte ich nachfolgend eine alternative Deutung an die Seite stellen. Ich werde zeigen, dass die derzeitige und mittelfristig absehbare Dynamik der europäischen Wirtschafts- und Sozialintegration maßgeblich von einem Umstand jenseits des demokratischen und sozialen Stands des Primärrechts determiniert wird: von der im Zeitverlauf zunehmenden politökonomischen Heterogenität der Mitgliedstaaten. Damit meine ich die Koexistenz höchst unterschiedlicher "Spielarten des Kapitalismus" in der EU-27. Diese Heterogenität beeinflusst unterschiedliche Integrationsdynamiken, namentlich die politische Integration und die judikative Integration, ${ }^{\underline{7}}$ auf asymmetrische Weise und entfaltet zudem Wirkungen auf die Funktionen und Dysfunktionen der gemeinsamen europäischen Währung. Die von der politökonomischen Heterogenität perpetuierte Asymmetrie zwischen politischer und judikativer Integration bewirkt einen wirtschaftsliberalen Bias der europäischen Integration, der auch dann nicht verschwinden würde, würden sich die politischen Kräfteverhältnisse in Europa nachhaltig zugunsten der Verfechter der Sozialen Demokratie verschieben. Mit dieser

Soziale Demokratie? Die politökonomische Heterogenität Europas als Determinante des demokratischen und sozialen Potenzials der EU (EuR-Bei 2013,69)

faktischen Eingrenzung europäischer Politikoptionen gehen deshalb auch Grenzen des Demokratiepotenzials der EU einher.

Um das Argument im Einzelnen zu entfalten, werde ich wie folgt vorgehen. In Abschnitt II. zeige ich die Heterogenität europäischer Wirtschafts- und Verteilungsregime anhand von Daten auf. In den drei darauf folgenden Abschnitten zeige ich, wie die Koexistenz heterogener "Spielarten des Kapitalismus" unterschiedliche Integrationsdynamiken und -potenziale entscheidend prägt. Dabei geht es um die Potenziale der politisch hergestellten Sozialintegration (III.), um die Dynamik der judikativen Integration (IV.) und um die Funktionsweise der europäischen Währungsunion (V.). Der abschließende Abschnitt VI. spitzt die Ergebnisse im Hinblick auf die Entwicklung einer europäischen Sozialen Demokratie zu und entwickelt die These, dass noch so wünschenswerte weitere Aufwertungen des EP kaum zusätzliches Demokratiepotenzial schöpfen.

\section{Die politökonomische Heterogenität der Europäischen Union}

Da das hier geführte Argument auf die Effekte der politökonomischen Heterogenität der EU zielt, werde ich diese Heterogenität zunächst anhand ausgewählter Daten illustrieren. Von Erweiterungsrunde zu Erweiterungsrunde hat die Unterschiedlichkeit der Wohlstandsniveaus zwischen den ursprünglich vergleichsweise homogenen Teilnehmern an der europäischen Integration zugenommen. Signifikante Zunahmen an Heterogenität ergaben sich insbesondere im Zuge der Süderweiterungen in den Jahren 1981 und 1986, im Zuge der Osterweiterung im Jahr 2004 und dann noch einmal im Zuge des Beitritts Bulgariens und Rumäniens im Jahr 2007. Bei der Analyse von Wohlstandsniveaus empfiehlt es sich, das Bruttoinlandsprodukt (BIP) pro Kopf in so genannten Kaufkraftstandards zu vergleichen, um Verzerrungen durch regionale Kaufkraftdivergenzen Rechnung zu tragen. So berechnet, erreichen fünf sehr wohlhabende Länder ein Wohlstandsniveau, das mehr als $20 \%$ über dem Durchschnitt liegt: Luxemburg, Irland, die Niederlande, Österreich und Schweden. Diesen stehen zehn Länder gegenüber, deren Wohlstandsniveau unter $80 \%$ des Durchschnitts liegt, darunter Portugal, Malta und acht der ehemals sozialistischen Transformationsländer. Die ärmsten EU-Mitglieder, Bulgarien und Rumänien, rangieren unterhalb der Hälfte des Durchschnitts der EU-27, während das polnische Wohlstandsniveau bei etwa 58 \% des

Durchschnitts liegt. ${ }^{8}$ Diese Wohlstandsniveaus reflektieren die unterschiedlichen Produktivitätsniveaus der beteiligten Volkswirtschaften und bilden sich deshalb auch in den Lohnniveaus ab, woraus manifeste Interessenkonflikte zwischen den Mitgliedstaaten resultieren als Beispiele seien die sehr heterogenen Interessen an sozialpolitischer Harmonisierung und an der Übertragung von arbeitsrechtlichen und lohnbezogenen Mindeststandards auf transnational entsandte Beschäftigte genannt. ${ }^{9}$

Soziale Demokratie? Die politökonomische Heterogenität Europas als Determinante des demokratischen und sozialen Potenzials der EU (EuR-Bei 2013,69)

Selbst wenn sich die Wohlstandsniveaus der EU-Länder rasch angleichen würden, blieben jene Unterschiede bestehen, die sich aus den organisatorischen und institutionellen Ausgestaltungen der beteiligten Produktions- und Verteilungsregime ergeben. In der Terminologie der Vergleichenden Politischen Ökonomie: Die Europäische Union besteht aus höchst unterschiedlichen "Spielarten des Kapitalismus". .100 Diese Organisationen und Institutionen und die in ihnen betriebenen Praktiken haben sich über lange Zeiträume entwickelt und verändern sich nur langsam. So entfallen ungefähr 12-14 \% des BIP in Estland, Lettland und Litauen auf Sozialschutzausgaben, aber um die $31 \%$ in Frankreich und Schweden. ${ }^{11}$ Hinter diesen Zahlen verbergen sich tiefer liegende Unterschiede: Die europäischen Wohlfahrtsstaaten sind nicht nur unterschiedlich generös, sondern folgen auch unterschiedlichen Strukturprinzipien. So finden wir die steuerfinanzierten Systeme Dänemarks und Irlands neben sozialversicherungsfinanzierten Systemen der Niederlande und Belgiens und Mischsystemen wie dem deutschen. Manche Lohnersatzleistungen sind auf den Statuserhalt im Falle der Arbeitslosigkeit ausgerichtet, andere auf Mindestabsicherungen. ${ }^{12}$ Als ähnlich heterogen erweisen sich beispielsweise die Institutionen und Praktiken der Unternehmenskontrolle: In einigen Ländern verfügen die Unternehmen über separate Aufsichtsräte, die die Vorstände einsetzen und kontrollieren, in anderen gibt es ein gemeinsames Leitungsorgan. In einige dieser Aufsichts- und Exekutivorgane werden Arbeitnehmervertreter entsandt, andere kennen keinerlei Arbeitnehmermitbestimmung auf Ebene der Leitungsorgane..$^{\underline{13}}$

Auch in Bezug auf die Verbände der Sozialpartner weisen die Mitgliedsländer der EU erhebliche Unterschiede auf. Die

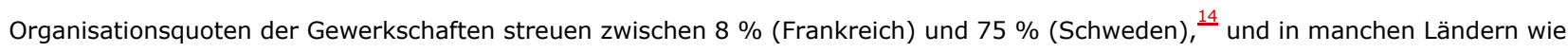


Österreich sind praktisch alle, in anderen wie Polen hingegen nur sehr wenige Unternehmen in Arbeitgeberverbänden organisiert. ${ }^{15}$ Die Deckungsgrade von Tarifverträgen variieren zwischen weniger als $20 \%$ (Litauen) und mehr als $90 \%$ (Belgien, Österreich, Schweden). ${ }^{16}$ Diese Beispiele illustrieren lediglich einen geringen Teil der Unterschiede zwischen den auf dem europäischen Kontinent vertretenen Politischen Ökonomien, zahlreiche weitere ließen sich nennen - vom Arbeitsrecht über die Aktienkulturen oder die Institutionen der Berufsbildung bis hin zur Verbreitung und Gestaltung von Betriebsrenten.

Die Vergleichende Politische Ökonomie hat eine große Anzahl an Vorschlägen zur Kategorisierung solcher "Spielarten des Kapitalismus" hervorgebracht. Manche

Soziale Demokratie? Die politökonomische Heterogenität Europas als Determinante des demokratischen und sozialen Potenzials der EU (EuR-Bei 2013, 69)

Autoren unterscheiden koordinierte und liberale Kapitalismen; $i^{17}$ andere liberale, konservative und sozialdemokratische Regime ${ }^{18}$ einige interpretieren die südeuropäische Ländergruppe als eigenständige "Spielart des Kapitalismus", ${ }^{19}$ andere wiederum spalten aus der Gruppe der eher liberalen Regime ein spezifisch osteuropäisches Modell ab..$^{20}$ Entscheidend ist an dieser Stelle: Welche Produktions - und Verteilungsregimes westlicher Industrieländer man auch immer unterscheidet, sie finden sich alle auf dem europäischen Kontinent. Mit anderen Worten: Die Spannweite der Strukturprinzipien der Politischen Ökonomien Europas ist gleichbedeutend mit der Spannweite, die wir aus der Grundgesamtheit der entwickelten Industrieländer kennen.

Daraus folgt zunächst zweierlei. Erstens, der Status Quo lässt kein spezifisch europäisches Produktionsregime und namentlich kein spezifisch europäisches Sozialmodell erkennen, und wenn doch, dann ist letzeres so vage, dass es gleichbedeutend ist mit dem Sozialen in den entwickelten Industrieländern. Und zweitens, die europäische Integration ist keine Integration unter Gleichen. Unter der begründeten Annahme, dass Regierungen institutionelle Anpassungslasten zu minimieren suchen, ist mit manifesten Interessenkonflikten zu rechnen, wenn Integrationsprojekte die institutionellen Grundpfeiler ihrer politökonomischen Regime tangieren. Ebenso manifeste Interessenkonflikte resultieren aus den in Europa vertretenen Produktivitäts- und Wohlstandsniveaus. Es wäre deshalb verwunderlich, würde die politökonomische Heterogenität der Mitgliedstaaten die Integrationsdynamiken nicht maßgeblich prägen. In den nachfolgenden Abschnitten gilt es, diese Dynamiken eingehender zu spezifizieren.

\section{Hemmnisse der politisch herbeigeführten Sozialintegration}

Eine europäische Sozialpolitik, die Verfechter der Sozialen Demokratie zufriedenstellen würde, setzt politische Akte voraus, denen durch das Nebeneinander heterogener mitgliedstaatlicher Interessen einerseits und eines mit zahlreichen Vetopunkten durchsetzten politischen Systems Europas andererseits aber enge Grenzen gesetzt sind. Die integrationstheoretischen Grundlagen zur Analyse dieser Konstellation haben Joseph Weiler mit seiner Unterscheidung zwischen intergouvernementaler politischer Integration und supranationaler Rechtsproduktion sowie Fritz Scharpf mit seinem Modell der Politikverflechtungsfalle und dessen Anwendung auf die europäische Wirtschafts- und Sozialintegration gelegt. ${ }^{21}$

Soziale Demokratie? Die politökonomische Heterogenität Europas als Determinante des demokratischen und sozialen Potenzials der EU (EuR-Bei 2013, 69)

Worin liegen die spezifischen Schwierigkeiten marktkorrigierender Integration? Die Übertragung von Kompetenzen an die europäische Ebene setzt Vertragsänderungen voraus, die stets nur unter Zustimmung aller Mitgliedstaaten erfolgen können; im Grenzfall genügt das Veto einzelner EU-Mitglieder, um entsprechende Vertiefungen der Integration zu verhindern. Aber auch wo die EU bereits über Kompetenzen verfügt und die Gemeinschaftsmethode Anwendung findet, ist die Ausfüllung der entsprechenden

Handlungsmöglichkeiten ein mühseliger Prozess, der einen Kommissionsvorschlag, eine einstimmige oder qualifizierte Mehrheit im Rat und eine ausreichende politische Mehrheit im EP voraussetzt. Wo mit qualifizierter Mehrheit entschieden werden kann, achten die Mitgliedstaaten darauf, die fundamentalen Interessen einzelner Ländergruppen nicht systematisch zu verletzten. Wo also die Einstimmigkeitsregel nicht mehr greift, wird gleichwohl zumindest Einvernehmlichkeit angestrebt. ${ }^{22}$ Dies legt Maßnahmen der marktkorrigierenden Integration hohe Hürden auf.

Freilich folgt hieraus nicht, Integrationsfortschritte seien in marktgestaltenden Politikfeldern ausgeschlossen. In zahlreichen Bereichen wie beispielsweise der Arbeitssicherheit, ${ }^{23}$ der Umwelthaftung ${ }^{\underline{24}}$ und dem Verbraucherschutz ${ }^{\underline{25}}$ ergingen Richtlinien, die das Regulierungsniveau in vielen Mitgliedstaaten spürbar erhöhten. Unter Moderation der Kommission gelang es den Mitgliedstaaten in diesen Feldern, Kompromisse zu finden und damit zu einheitlichen Regelungen zu gelangen, die die Effektivität des gemeinsamen Binnenmarkts erhöhten. Die genannten Regelungsbereiche haben allerdings gemeinsam, dass die Interessen der Mitgliedstaaten nicht systematisch zwischen unterschiedlichen „Spielarten des Kapitalismus" variierten. Die Länder sahen sich vielmehr vergleichbaren Situationen ausgesetzt, weil die Konfliktlinien zuvörderst innerhalb der Länder verliefen und gemeinsame Lösungen durch die Effektuierung des Binnenmarkts Zugewinne für alle versprachen. In solchen Situationen hängen Kompromisse vom politischen Willen zur Schöpfung europäischer Lösungen, vom Verhandlungsgeschick der Kommission und von der Verfügbarkeit von Paketlösungen ab. ${ }^{26}$ Anders stellt sich die Situation aber dar, wo eine etwaige europäische Harmonisierung divergierende Länderinteressen so fundamental berührt, dass die betreffenden Mitgliedstaaten befürchten müssen, am Ende als entweder Verlierer oder Gewinner dazustehen, entweder aufgrund befürchteter Wohlstandstransfers oder aufgrund asymmetrischer institutioneller Anpassungslasten. ${ }^{27}$ Diese Beschreibung kommt den Interessenkonstellationen in all jenen Politikbereichen nahe, die zu den Kernelementen der "Spielarten des Kapitalismus" zählen. Anschauliche Beispiele für solch „integrationsresistente" Bereiche sind die Generosität und Beschaffenheit der historisch gewachsenen Systeme sozialer Sicherung, die Modalitäten der 
Soziale Demokratie? Die politökonomische Heterogenität Europas als Determinante des demokratischen und sozialen $\boldsymbol{\nabla}$ Potenzials der EU (EuR-Bei 2013, 69)

Lohnaushandlung und die Arbeitnehmermitbestimmung auf Ebene der Leitungsorgane von Unternehmen. Nichts anderes gilt für die Besteuerung von Kapital und Arbeit.

Mit der gestiegenen Heterogenität der in der EU vertretenen Produktivitäts- und Regulierungsniveaus haben auch die Interessensunterschiede zugenommen, die überbrückt werden müssen, um zu einvernehmlichen Lösungen zu gelangen. Erscheinen Verlagerungen auf die europäische Ebene aufgrund tiefgreifender Interessenunterschiede ausgeschlossen, bleiben autonomieschonende Lösungen, die die mitgliedsstaatlichen Praktiken vor europäischen Zugriffen schützen, die für alle Beteiligten verträglichste Option. So ist es kein Zufall, dass die Mitgliedstaaten gerade für die sensiblen Bereiche der Lohnfindung und des Arbeitskampfrechts eine explizite Sperrklausel in die europäischen Verträge aufgenommen haben, um sie von den sozialpolitischen Kompetenzen der EU auszunehmen (Art. $\underline{153}$ Abs. $\underline{5}$ AEUV).

Nur folgerichtig ist, dass die Schaffung eines vereinheitlichten europäischen Sozialstaats weder auf der europäischen Tagesordnung steht, noch auch nur grundsätzlich für sinnvoll gehalten wird. Die Funktionen europäischer Sozialpolitik erschöpfen sich vielmehr in der (vor allem in den neunziger Jahren erfolgten) Etablierung von für alle akzeptablen Mindeststandards in Bereichen wie dem Recht auf Elternurlaub ${ }^{28}$ und der Arbeitszeit ${ }^{29}$ sowie in der weichen, transnationalen Koordinierung der nationalen sozialpolitischen Praktiken. Die erstmals 1993 zur Koordination von Arbeitsmarktpolitiken eingeführte "Offene Methode der Koordinierung" (OMK) setzt auf unverbindliche Leitlinien und Empfehlungen, auf gegenseitiges Lernen und die Beobachtung und Dokumentation der nationalen Politiken durch die Kommission und die europäische Statistikbehörde Eurostat. ${ }^{30}$ Ob durch die OMK Kurswechsel in den nationalen Sozialpolitiken angestoßen wurden, wird in der Fachliteratur skeptisch beurteilt. ${ }^{31}$

Die Diagnose einer durch die politökonomische Heterogenität systematisch gehemmten sozialpolitischen Integration in der EU-27 ist noch nicht notwendigerweise eine schlechte Nachricht für die Soziale Demokratie, denn bis hier verweist die Argumentation lediglich auf die gebremste Geschwindigkeit, in der die Schaffung eines europäischen Sozialraums überhaupt nur vonstatten gehen kann. Eine bedenkliche Schlagseite gewinnt die europäische Integration hierdurch nicht. Denn vor genau denselben Schwierigkeiten steht schließlich auch die marktschaffende Integration, die sich ebenfalls immer wieder blockiert sieht, sobald die Mitgliedstaaten fundamentale nationale Interessen berührt sehen, weil sie transnationale Verteilungseffekte oder problematische institutionelle Anpassungserfordernisse befürchten. Das haben insbesondere die politisch aufreibenden Auseinandersetzungen

Soziale Demokratie? Die politökonomische Heterogenität Europas als Determinante des demokratischen und sozialen Potenzials der EU (EuR-Bei 2013, 69)

um die Übernahmerichtlinie ${ }^{\frac{32}{2}}$ und die Dienstleistungsrichtlinie $e^{\frac{33}{3}}$ gezeigt. Solange auf europäischer Ebene keine Strukturen entstehen, die den Kriterien einer Sozialen Demokratie gerecht würden, existieren auf Ebene der Mitgliedstaaten mehr oder minder ausgeprägte Soziale Demokratien, die zunehmend, wenn auch langsam, um eine zusätzliche Flankierung des Sozialen auf europäischer Ebene ergänzt werden - so ließe sich argumentieren. Alarmierend wird die Diagnose aber, wenn bedacht wird, dass neben der Integration durch Politik eine weitere Integrationsdynamik existiert, die ebenfalls Wirkungen auf das Soziale entfaltet, die nicht durch die Heterogenität der Mitgliedstaaten gebremst wird und die selbst die zum Autonomieschutz installierten Sperrklauseln wie in Art. 153 Abs. $\underline{5}$ AEUV zunehmend durchstößt: die judikative Integration, der ich mich nachfolgend zuwende.

\section{Die Wucht der judikativen Integration}

Im Unterschied zur durch die Heterogenität der Mitgliedstaaten auf mittlere Sicht verunmöglichten Schaffung eines europäischen Sozialstaats profitieren marktschaffende Projekte von der Dynamik der judikativen Integration: von der extensiven Auslegung der wettbewerbspolitischen Kompetenzen der Kommission und insbesondere von der EuGH-Rechtsprechung zu den europäischen Grundfreiheiten. In der rechts- und sozialwissenschaftlichen Literatur zum EuGH ist unumstritten, dass das europäische Höchstgericht im europäischen Integrationsprozess nicht lediglich als „Hüter der Verträge" wirkte. Vielmehr und darüber hinaus betrieb der EuGH von Beginn an eine aktivistische, proeuropäische Rechtsprechung und wurde so zum "Motor der Integration". . $^{4}$

Eine besondere Brisanz kommt hierbei der Grundfreiheiten-Judikatur des EuGH zu, weil Ausweitungen des Anwendungsbereichs der Grundfreiheiten aufgrund des Vorrangs und der Direktwirkung des Unionsrechts geeignet sind, nationales Recht unterschiedlichster Regelungsbereiche ohne Zutun der Politik zu verdrängen. Hierzu wendet der EuGH seit seinem Gebhard-Urteil von 1995 den in der Dassonville- und Cassis de Dijon-Rechtsprechung entwickelten vierstufigen Test einheitlich auf alle Grundfreiheiten an: Beschränkungen der transnationalen Ausübung von Grundfreiheiten sind nur dann europarechtsform, wenn sie aus zwingenden Gründen des Allgemeininteresses gerechtfertigt sind, in nichtdiskriminierender Weise angewandt werden, zur Erreichung des als zwingend anerkannten Ziels tatsächlich geeignet sind und über das zur Zielerreichung Notwendige nicht hinausgehen. $\frac{35}{1}$ Mit seiner extensiven Interpretation der europäischen Grundfreiheiten greift der EuGH mittlerweile tief in sensible Bereiche mitgliedsstaatlicher Gestaltungshoheit ein, und zwar auch dann, wenn für die entsprechenden Bereiche keine

Soziale Demokratie? Die politökonomische Heterogenität Europas als Determinante des demokratischen und sozialen Potenzials der EU (EuR-Bei 2013, 69)

Regelungskompetenzen der EU bestehen oder sie durch Regelungsvorbehalte wie dem Art. $\underline{153}$ Abs. $\underline{5}$ AEUV vor dem politischen Zugriff der Unionsorgane geschützt sind. $\frac{36}{,}, \underline{37}$ Entscheidend für die vorliegende Argumentation ist nun, dass die extensive Rechtsprechung zum Anwendungsbereich der Grundfreiheiten dazu führt, dass marktkorrigierende Regelungen auf mitgliedsstaatlicher Ebene vor dem EuGH - wie Eva Kocher sagt - „regelmäßig rechtfertigungsbedürftig” und entsprechend prekär werden. ${ }^{38}$ Hinzu kommt, 
dass es nach der mit Viking und Laval begonnenen Linie der Rechtsprechung hierfür keinen Unterschied macht, ob die Gegenstände dieser Rechtfertigungspflicht ein vom EuGH anerkanntes Grundrecht tangieren. ${ }^{39}$ Nur folgerichtig ist dann, dass auch die mit dem Vertrag von Lissabon erreichte Aufwertung der Grundrechte-Charta für die Grundfreiheiten-Judikatur des EuGH keinen erkennbaren Unterschied macht. ${ }^{40}, \underline{41}$ Die nach der Aufwertung der Grundrechte-Charta ergangene EuGH-Entscheidung zur betrieblichen Altersvorsorge kommunaler Arbeitgeber hat dies eindrucksvoll bestätigt. ${ }^{42}$ Kurz, die ursprünglich als Diskriminierungsverbote gedachten europäischen Grundfreiheiten wirken als höchst wirksame Instrumente wirtschaftlicher Liberalisierung, unbeschadet von autonomieschonenden Regelungsvorbehalten im Primärrecht oder europäischen sozialen Grundrechten.

Dieser Form der judikativen Intervention legt die politökonomische Heterogenität der Mitgliedstaaten keine Hürden auf. Ganz im Gegenteil: Die politökonomische Heterogenität Europas erweitert die Spielräume aktivistischer Rechtsprechung, schon weil sie die legislative Korrektur politisch unerwünschter Judikate erschwert. Diese These wird bestätigt aus den Einsichten der vergleichenden Forschung über Verfassungsgerichte. Verfassungsrichter, so der Diskussionsstand in dieser Literatur, operieren nicht in luftleerem Raum. Vielmehr bedenken sie mögliche

Soziale Demokratie? Die politökonomische Heterogenität Europas als Determinante des demokratischen und sozialen Potenzials der EU (EuR-Bei 2013, 69)

Rückwirkungen ihrer Rechtsprechung auf die Teilautonomie der betreffenden Höchstgerichte. Um ihre Unabhängigkeit nicht zu gefährden, sind sie bemüht, die Wahrscheinlichkeit der nachgelagerten politischen Korrektur ihrer Urteile zu minimieren. ${ }^{43}$ Im politischen System Europas sind solche Korrekturen allerdings höchst unwahrscheinlich - was den Spielraum des europäischen Höchstgerichts, integrationsvertiefende Rechtsfortbildung zu betreiben, entsprechend maximiert. ${ }^{44}$ Denn die Hürden, die der Korrektur von EuGH-Urteilen aufliegen, sind identisch mit den in Abschnitt III. behandelten Hürden politischer Integration.

In Abschnitt III. habe ich unter Verweis auf die Arbeiten von Scharpf herausgestellt, dass die der politischen Integration aufliegenden Hürden hoch, aber keineswegs unüberwindlich sind, insbesondere dort, wo durch geschickte Verhandlungsführung der Kommission Kooperationsgewinne erwirtschaftet werden können. Hingegen sinkt die Wahrscheinlichkeit zu einer Einigung zu gelangen, wenn das Ergebnis stets Gewinner und Verlierer hinsichtlich Wohlstandstransfers und asymmetrischen institutionellen Anpassungslasten hervorbringen muss. ${ }^{45}$ Eben dies ist aber häufig der Fall, wenn es um die Grundfreiheiten-Judikatur des EuGH geht, die stets auf die eine oder andere Weise die Arbeitsteilung zwischen Staat (und anderen Formen kollektiver Regulierung) und Markt in den heterogenen "Spielarten des Kapitalismus" der EU-27 tangiert. Drohungen mit nachgelagerten politischen Korrekturen sind vor diesem Hintergrund unglaubwürdig und können vom europäischen Höchstgericht ignoriert werden. Dieselbe Logik greift für alle anderen Formen des "court curbing", die in der vergleichenden Literatur über Verfassungsgerichte als Mechanismen der politischen Disziplinierung identifiziert wurden, wie zum Beispiel Eingriffen in die Zuständigkeiten des Höchstgerichts, Drohungen mit finanziellen Einschnitten oder anderen institutionellen Reformen der Gerichtsbarkeit. ${ }^{46}$

Nun ist einzuräumen, dass sich die "aktivistische", pro-europäische Rechtsprechung des EuGH keineswegs auf die extensive Interpretation des Anwendungsbereichs der Grundfreiheiten (sowie einer ebenso extensiven Interpretation des Anwendungsbereichs des europäischen Wettbewerbsrechts) beschränkt. Mindestens zwei weitere Linien der Rechtsprechung sind für die Soziale Demokratie von Belang. ${ }^{47}$ Erstens, der EuGH hat eine extensive Antidiskriminierungs-Rechtsprechung zum Schutz des Einzelnen vor Diskriminierungen aufgrund des Alters, des Geschlechts, der sexuellen Orientierung und der Zugehörigkeit zu Ethnien und Religionen entwickelt. Obgleich diese Stärkung des individuellen Schutzes vor diskriminierenden (staatlichen oder privaten) Praktiken nicht mit der Schaffung eines europäischen

Soziale Demokratie? Die politökonomische Heterogenität Europas als Determinante des demokratischen und sozialen Potenzials der EU (EuR-Bei 2013,69)

Sozialstaats verwechselt werden darf, so haben diese Urteile gleichwohl einen sozialen Gehalt. ${ }^{48}$ Die "neoliberale" Rechtsprechung des EuGH findet also, wie Scharpf es ausdrückt, eine Ergänzung durch eine "sozialliberale" Gleichstellungsjudikatur. ${ }^{49}$ Zweitens, in einer langen Serie von Urteilen hat der EuGH die transnationale Öffnung der sozialen Sicherungssysteme, einschließlich der Gesundheitssysteme, vorangetrieben und damit die sozialen Rechte zunächst von Arbeitsmigranten, zunehmend aber auch aller Unionsbürger unabhängig von der Ausübung einer Erwerbstätigkeit gestärkt. ${ }^{50}$ Ob diese Rechtsprechung als Keimzelle einer in Entstehung begriffenen transnational-europäischen Solidarität wirkt, oder aber vor allem De-regulierungsdruck auf die überdurchschnittlich generösen Wohlfahrtsstaaten erzeugt, ist allerdings umstritten. ${ }^{\underline{51}}$

Während also die Hemmnisse der sozialen Integration für sich genommen noch nicht notwendigerweise problematisch erscheinen, bewirkt die Gleichzeitigkeit von gebremster politischer Integration und stetig voranschreitender judikativer Integration eine asymmetrische Integrationsdynamik, die dem Integrationsprojekt - unabhängig von den Mehrheitsverhältnissen im EP und im Rat einen spezifischen politökonomischen Drall verleiht und in Richtung einer mit "linksliberalen" Elementen angereicherten wirtschaftlichen Liberalisierung wirkt. Dies konstituiert, wie ich im abschließenden Abschnitt VI. argumentieren werde, nicht nur ein Problem für die Potenziale des Sozialen auf europäischer Ebene, sondern auch für die Demokratie. Vorher aber wende ich mich einem weiteren Bereich zu, der ebenfalls nachhaltig durch die Koexistenz heterogener europäischer "Spielarten des Kapitalismus" geprägt wird, und zwar der europäischen Währungsunion.

\section{Die europäische Währungsunion vor dem Hintergrund politökonomischer Heterogenität}

Die Europäische Zentralbank (EZB) sieht sich der Herausforderung gegenüber, auf höchst heterogene Wirtschaftsverläufe in der Eurozone mit ein und demselben nominalen Zinssatz reagieren zu müssen. Der Euro-Raum ist kein einheitlicher Konjunkturraum und 
war es von Anfang an nicht. In lediglich zwei der zehn Jahre zwischen der Einführung des Euro-Buchgelds (1999) und dem Ausbruch der Finanzkrise (2008) lag die deutsche Inflationsrate oberhalb des EZB-Inflationsziels von zwei Prozent. In Irland, Griechenland, Portugal und Spanien hingegen lag die

Soziale Demokratie? Die politökonomische Heterogenität Europas als Determinante des demokratischen und sozialen Potenzials der EU (EuR-Bei 2013, 69)

Inflationsrate in allen zehn Jahren oberhalb der von der EZB anvisierten Zielinflation. $\frac{52}{B i s}$ zu drei Prozentpunkte und manchmal sogar etwas mehr lagen die Inflationsraten Deutschlands einerseits, Portugals und Irlands andererseits in den frühen 2000er Jahren auseinander, eine ähnliche Varianz wiesen die europäischen Wachstumsraten auf. Die Folge: Mit demselben Nominalzins musste die EZB sowohl die deutsche Stagnation als auch die irische und südeuropäische Inflation bekämpfen. Der gewählte Zinssatz musste für Deutschland zu hoch, für alle anderen genannten Länder zu niedrig ausfallen - es konnte gar nicht anders sein.

Damit nicht genug. Heizte ein zu niedriger Nominalzins die Wirtschaft der Inflationsländer zusätzlich an, dann sanken die Realzinsen immer weiter (denn die Realzinsen sind definiert als Nominalzinsen abzüglich der Inflationsrate). Konjunktur und Inflation wurden durch dysfunktional niedrige Zinssätze stimuliert, obwohl eine stimmige Geldpolitik eigentlich zur Abkühlung der Konjunktur hätte beitragen müssen - ein Effekt, der namentlich auch die Entstehung und das Wachstum der spanischen und irischen Immobilienblasen begünstigte. Und wurde ein Land mit einem zu hohen Nominalzins konfrontiert und sank deshalb die Inflation, dann stieg der Realzins, obwohl er doch eigentlich hätte fallen müssen, um zur Entspannung der wirtschaftlichen Lage beizutragen. Trifft also eine einheitliche Geldpolitik auf heterogene Entwicklungen der Preisniveaus, dann macht sie die eigentlich ja angestrebte Konvergenz wirtschaftlicher Entwicklungen schwerer, nicht etwa leichter. $\underline{\underline{53}}$

Mit welchen Instrumenten nun lässt sich diesem Problem eines geldpolitisch begünstigten Auseinanderdriftens europäischer Konjunkturverläufe entgegenwirken? Die Geldpolitik fällt als Hebel aus, und auch der Fiskalpolitik setzt der Stabilitäts- und Wachstumspakt Grenzen. Die einheitliche Geldpolitik potenziert deshalb die Anforderungen an eine funktionale, makroökonomischen Bedingungen Rechnung tragende, vorausschauend agierende Lohnpolitik. Und hier kommen die heterogenen "Spielarten des Kapitalismus" der Eurozone ins Spiel. Denn wie hat sich Deutschland aus der misslichen Lage eines unangemessen hohen, die deflationären Tendenzen zusätzlich verstärkenden Realzinses befreit? Durch eine lange Serie höchst moderater Lohnabschlüsse und den damit einhergehenden gezielten Aufbau von Exportüberschüssen, also nicht zuletzt: zu Lasten der europäischen Nachbarn. Seit der Euro-Einführung türmt Deutschland trendmäßig steigende Handelsbilanzüberschüsse auf, die seit 2004 durchgängig eine Größenordnung von um die fünf Prozent des BIP erreichen. Im Jahr 2009 betrug der deutsche Überschuss 4,6 \%, im Jahr zuvor sogar $6,3 \%$.

Entscheidend ist nun, dass die Fähigkeit zu strategisch herbeigeführter Lohnzurückhaltung über unterschiedliche "Spielarten des Kapitalismus" unterschiedlich verteilt ist. Zum gesicherten Forschungsstand der Vergleichenden Politischen Ökonomie zählt die Einsicht, dass unterschiedliche Systeme der Lohnfindung

Soziale Demokratie? Die politökonomische Heterogenität Europas als Determinante des demokratischen und sozialen Potenzials der EU (EuR-Bei 2013, 69)

unterschiedliche Nominallohnauftriebe hervorbringen. ${ }^{54}$ Ausgeprägt ist die Fähigkeit zur strategischen Lohnzurückhaltung in hoch koordinierten Systemen der Arbeitsbeziehungen, also in zentralisierten Systemen wie den skandinavischen oder etwa im deutschen, das zwar formal nicht zentralisiert ist, in dem aber sektorale und regionale Lohnabschlüsse mit ausgeprägter Signalwirkung auf einen hohen Grad an Koordination hinwirken. Euro-Länder mit dezentralen, unkoordinierten Formen der Lohnaushandlung, vor allem in Südeuropa, haben dem nichts entgegenzusetzen. Ihre preisliche Wettbewerbskraft schwindet, ohne dass die Möglichkeit einer nominalen Abwertung der Währung als Anpassungsinstrument zur Verfügung stünde. In den Jahren 2008 und 2009 betrugen die Handelsbilanzdefizite Spaniens 5,6 \% bzw. 2,1 \%, die Salden Portugals 9,6 \% bzw. 7,6 \% und die Defizite Griechenlands 10,3\% bzw. $9,7 \%$. Auch die französische Handelsbilanz weist seit Mitte der 2000er Jahre durchgehend Defizite auf, ebenso wie - wenn auch weniger ausgeprägt - die italienische Handelsbilanz. Im Euro-Raum besteht nunmehr ein deutlicher statistischer Zusammenhang zwischen der Gewerkschaftsstärke einerseits und den Salden der Handelsbilanz andererseits: Wo die Gewerkschaften stark sind und die Lohnfindungssysteme koordiniert, wie es neben Deutschland etwa in Österreich, Finnland und den Niederlanden der Fall ist, werden Exportüberschüsse erzielt.

Die derzeitige Konstellation lässt vermuten, dass nun die deutsche Konjunktur für einige Jahre von einem zu niedrigen Realzins begünstigt werden dürfte, der für Länder wie Griechenland und Spanien aber gleichwohl zu hoch ausfallen muss. Der Unterschied zum Zyklus der ersten Euro-Jahre ist nur, dass den Südeuropäern der deutsche Weg der Problembewältigung - gezielte Lohnzurückhaltung gegenüber den anderen Euro-Ländern und damit letztlich beggar my neighbour - nicht zur Verfügung stehen wird. Die einheitliche Geldpolitik läuft somit Gefahr, vor dem Hintergrund systematisch divergenter Lohnauftriebe die preisliche Wettbewerbsstärke der EuroTeilnehmerländer nachhaltig zu Gunsten der koordinierten und zu Ungunsten der unkoordinierten Länder zu verschieben. Diese Ungleichgewichte sind das Ergebnis eines im Euro strukturell eingepflanzten Konstruktionsproblems, für das kurz- bis mittelfristig keine Lösung in Sicht ist. Koordinierte Lohnaushandlungssysteme sind über lange historische Zeiträume gewachsen. Die in den vergangenen zehn Jahren zahlreichen Versuche der südeuropäischen und irischen Regierungen, im Rahmen so genannter Sozialer Pakte auf Lohnmoderation hinzuwirken, sind weitgehend gescheitert. ${ }^{55}$ Eine europäisch koordinierte Lohnpolitik scheint kurz- bis mittelfristig ebenso undurchsetzbar wie eine Transferunion, die nicht auf

Soziale Demokratie? Die politökonomische Heterogenität Europas als Determinante des demokratischen und sozialen Potenzials der EU (EuR-Bei 2013,69) 
Zu vermuten ist, dass die Funktionsweise des Euro - ebenso wie die in den Abschnitten III. und IV. hergeleitete Asymmetrie zwischen politischer und judikativer Integration - auf lange Sicht Deregulierungsdruck induziert, deshalb die Politikoptionen einschränkt und sie, selbst wenn dies von den politischen Mehrheitsverhältnissen eigentlich nicht gedeckt ist, in eine spezifische Richtung lenkt. Treiben die Dysfunktionen des Euro die Makroökonomien der Teilnehmerländer auseinander, wird die europäische Ebene mit Druck zur lohn- und haushaltspolitischen Gegensteuerung reagieren müssen. Dieser Druck müsste theoretisch einige Länder zu restriktiver, andere Länder - namentlich jene des alten „DM-Blocks" und also vor allem Deutschland - zu expansiver makroökonomischer Politik anhalten. Weil aber unklar ist, mit welchen Mechanismen Länder dazu angehalten werden könnten, sich lohn- und fiskalpolitisch expansiver zu verhalten als von ihnen gewünscht, erscheint nur der Druck in Richtung restriktiverer Politik eine realistische Option. Hier wiederum kann der Druck auf die Lohnpolitik nur die Form weicher Appelle annehmen. Es bleibt die Fiskalpolitik, die harten, sanktionierbaren Regeln (wie jenen des Stabilitätspakts und künftiger Regeln haushaltspolitischer Überwachung im Euro-Raum) ${ }^{\frac{56}{6}}$ unterworfen werden kann. Meine These ist deshalb, dass die Währungsunion auf lange Sicht einen systematischen Druck zur Ausgabenmoderation und damit zur Senkung der Staatsquoten erzeugt. Weil die wesentliche Determinante unterschiedlicher Staatsquoten die Generosität der Wohlfahrtsstaaten ist, ${ }^{57}$ ist dieser Druck gleichbedeutend mit Druck auf den Sozialstaat. Für Verfechter der Sozialen Demokratie kann auch dies keine gute Nachricht sein.

\section{Fazit: Drei Implikationen für die Soziale Demokratie in Europa}

Die Anhänger der Sozialen Demokratie sehen die Demokratie in doppelter Weise mit der sozialen Frage verknüpft: Das Soziale darf dem demokratischen Prozess nicht entzogen werden, und ein Mindestmaß an gleichberechtigtem Ressourcenzugang fungiert als Bestandsvoraussetzung einer lebendigen Demokratie. Ein im Sinne der Sozialen Demokratie funktionales politisches System ist daher durch seine Steuerungsfähigkeit gegenüber einer ihrerseits steuerbaren Ökonomie gekennzeichnet, und gute Politik hat, will sie ihren demokratischen Gehalt bewahren und ausbauen, eine soziale Zielsetzung. Wie ist es um Bestand und Chancen einer so verstandenen Sozialen Demokratie in der Europäischen Union bestellt? Vordergründig

Soziale Demokratie? Die politökonomische Heterogenität Europas als Determinante des demokratischen und sozialen Potenzials der EU (EuR-Bei 2013, 69)

84

hat sich die EU mit dem Vertragswerk von Lissabon den Idealvorstellungen einer europäischen Sozialen Demokratie weiter angenähert. Die Stärkung des EP verspricht einen Zugewinn an Demokratie, und auch der soziale Auftrag an das Handeln der europäischen Organe wurde gestärkt. Im vorliegenden Beitrag habe ich die Diskussion über die europäische Soziale Demokratie um einen Gesichtspunkt jenseits der primärrechtlichen Ausgestaltung des politischen Systems Europas erweitert: um die Wirkungen der von Erweiterungsrunde zu Erweiterungsrunde gestiegenen politökonomischen Heterogenität der Mitgliedstaaten. Die Argumentation führte zu einem von dem vordergründigen Eindruck abweichenden Ergebnis. Trotz aller Fortschritte im Primärrecht sind die Potenziale Sozialer Demokratie auf europäischer Ebene mit zunehmender Skepsis zu beurteilen.

Der Kern des Problems liegt in den asymmetrischen Wirkungen der politökonomischen Heterogenität Europas auf unterschiedliche Integrationsdynamiken: auf die Chancen des Transfers marktkorrigierender Arrangements auf die europäische Ebene einerseits und auf die Wucht der marktschaffenden judikativen Integration andererseits. Angesichts der zunehmenden Heterogenität von Wohlstandsniveaus und institutionellen Ausgestaltungen der mitgliedsstaatlichen Produktions- und Verteilungsregime erscheinen politisch herbeigeführte Harmonisierungen der europäischen Sozialstaaten auf mittlere Sicht ausgeschlossen. Die Hoffnungen auf langfristige Fortschritte ruhen deshalb vor allem auf der weichen, unverbindlichen Koordinierung. Dieser Umstand allein wäre nicht alarmierend, würde der politischen Integration nicht eine weitere Integrationsdynamik gegenüberstehen, die ebenfalls auf das Soziale einwirkt: die judikative Integration, die durch die Interpretation und Durchsetzung europäischen Primär- und Sekundärrechts durch Kommission und EuGH zustande kommt. Da sich nahezu alle arbeits- und sozialrechtlichen Regelungen und Praktiken der Mitgliedstaaten letztlich als Beschränkungen der durch die EuGH-Rechtsprechung konstitutionalisierten Grundfreiheiten interpretieren lassen und die Grundfreiheiten-Judikatur immer mehr in sensible Kernbereiche mitgliedstaatlicher Autonomie eingreift, werden diese Regelungen und Praktiken zunehmend vor dem EuGH rechtfertigungsbedürftig. Erst diese Parallelität sozialer Implikationen sowohl der gehemmten politischen Integration als auch der Wucht der judikativen Integration verletzt die Bestandsvoraussetzungen Sozialer Demokratie. Sie entzieht die betroffenen Sachverhalte tendenziell dem Zugriff der Politik, untergräbt damit die politische Steuerungsfähigkeit und verleiht der europäischen Integration einen marktschaffenden, also wirtschaftsliberalen Drall.

Auf gleicher Augenhöhe begegnen sich europäische Politik und europäisches Recht hierbei nur in der Theorie. Im Prinzip steht es den Mitgliedstaaten offen, aus ihrer Sicht unangemessene Ausweitungen des Anwendungsbereichs der Grundfreiheiten durch Vertragsänderungen oder durch norminterpretierende Sekundärrechtssetzung zu korrigieren (gleiches gilt für weite Auslegungen des europäischen Wettbewerbsrechts). Tatsächlich aber macht das Zusammenspiel des Einstimmigkeitserfordernisses bei Vertragsänderungen und der heterogenen Interessenlagen der europäischen "Spielarten des Kapitalismus" nachgelagerte politische Korrekturen der

Soziale Demokratie? Die politökonomische Heterogenität Europas als Determinante des demokratischen und sozialen Potenzials der EU (EuR-Bei 2013,69)

Grundfreiheiten-Judikatur des EuGH so unwahrscheinlich, dass diese "Gefahr" vom europäischen Höchstgericht ignoriert werden kann. Es ist also die Heterogenität der in der EU vertretenen Produktions- und Verteilungsregime, die die Asymmetrie zwischen den Integrationsdynamiken dauerhaft perpetuiert und die europäische Wirtschafts- und Sozialintegration mit einem nachhaltigen liberalisierenden Bias versieht. $\stackrel{58}{ }$ Ein ähnlicher Effekt zeigte sich bei der Analyse der Funktionsweise der Währungsunion: Die Heterogenität der im Euro-Raum vertretenen "Spielarten des Kapitalismus" begünstigt die Entstehung makroökonomischer Ungleichgewichte, auf die die europäische Ebene reagieren muss. Weil sanktionierbare europäische Regeln nur zur Durchsetzung

https://beck-online.beck.de/default.aspx?typ=reference \&y=300\&z=EUR-BEI\&h=1\&b... 07.05 .2014 
vermehrter Haushaltsdisziplin in Ländern mit Exportdefiziten realistisch erscheinen, nicht aber zur Durchsetzung expansiverer Fiskalpolitik in Ländern mit Exportüberschüssen, wird im Ergebnis ebenfalls Deregulierungsdruck erzeugt.

Diese Einsichten haben weit reichende integrationstheoretische und -praktische Implikationen. Drei von ihnen seien angesprochen. Erstens, die politökonomische Heterogenität Europas macht die Entstehung eines europäischen Parteiensystems unwahrscheinlich und verdient deshalb, in der Debatte über die Demokratiefähigkeit Europas beachtet zu werden. Über die Fachgrenzen hinweg wurde darauf hingewiesen, dass sich die Demokratisierung des politischen Systems Europas durch eine Anzahl von Erschwernissen behindert sieht: durch das Fehlen eines sich als solchem begreifenden kollektiven europäischen Subjekts, durch die nationale Basiertheit politischer Diskurse, durch das Fehlen europäischer Medien, durch das Ausbleiben gemeinsamer Problemwahrnehmungen, durch die Nichtentstehung von Erinnerungs- und Erfahrungsgemeinschaften und, zuvörderst, durch das Fehlen einer gemeinsamen Sprache, die - über die europäischen Eliten hinausgehend - einen transnational-europäischen Diskurs überhaupt erst entstehen lassen könnte. ${ }^{59}$ All dies hemmt die Entwicklung der derzeit noch lockeren europäischen Parteienbündnisse zu europäischen Parteien, die sich auf kohärente politische Programme verständigen und diese dann gegenüber den Bürgerinnen und Bürgern zur Wahl stellen könnten.

Dieser Umstand wird kaum bestritten. ${ }^{60}$ Bei den Teilnehmerinnen und Teilnehmern der Debatte über das europäische Demokratiedefizit variiert aber die Intensität der Hoffnung auf die schrittweise Beseitigung dieser Hemmnisse transnational-europäischer Willensbildung etwa durch eine verstärkte europäische Berichterstattung der Medien, durch die gezielte Vermittlung von Kenntnissen über das politische System der EU und durch die zwar langsam erfolgende, aber doch stetige

Soziale Demokratie? Die politökonomische Heterogenität Europas als Determinante des demokratischen und sozialen Potenzials der EU (EuR-Bei 2013,69)

Überwindung von Kommunikationsbarrieren innerhalb der europäischen Parteienfamilien. ${ }^{\underline{61}}$

Die in dieser Literatur identifizierten Barrieren der Entstehung eines europäischen Parteiensystems existieren zweifellos. Das bedeutet aber nicht, dass das beklagte Problem verschwinden würde, wären die Barrieren beseitigt. Vereinfacht sei angenommen, dass sich die Präferenz einer politischen Gruppe gegenüber einer politökonomisch brisanten europäischen Richtlinie (oder eines vergleichbaren Rechtsakts wie z. B. einer EuGH-Entscheidung) aus zwei Quellen speist: einem ideologischen Interesse, das sich aus der Verortung der Gruppe auf der Links-rechts-Achse ergibt, und einem nationalen Interesse, das sich aus den durch die Maßnahme zu erwartenden transnationalen materiellen Verteilungswirkungen sowie den asymmetrischen institutionellen Anpassungslasten herleitet. Dann hätte die Entstehung europäischer Parteien mit kohärenten ideologischen Programmen zur Mindestvoraussetzung, dass die erste Quelle hinsichtlich ihrer Prägekraft über die zweite Quelle dominiert. Diese Voraussetzung wäre in einer Gruppe relativ homogener Länder gegeben, es darf aber bezweifelt werden, dass sie in der heterogenen EU der 27 Mitglieder mittelfristig erreichbar ist. ${ }^{62}$ Vor allem aber wird die Erfüllung dieser Voraussetzung sowohl von Erweiterungsrunde zu Erweiterungsrunde als auch in dem Maß, in dem die europäische Politik und das europäische Recht in politisch immer sensiblere Bereiche nationaler Autonomie eingreifen, unwahrscheinlicher. Das eigentliche Problem sind also wohl nicht die zahlreichen kulturellen Beschränkungen, Sprachprobleme und technischen Schwierigkeiten innerparteilicher Interessenaggregation und Abstimmungsprozesse. Nicht zuvörderst Kommunikationshemmnisse hindern die europäischen Parteien an der Herausbildung erkennbarer politischer Programme. Die heterogenen Interessenlagen sind vielmehr real und lassen sich von den Politikern, wollen sie wiedergewählt werden, nicht ignorieren - der politökonomische Status Quo determiniert nun einmal, welche Präferenzen politische Akteure gegenüber europäischen Entscheidungen vernünftigerweise haben können. So saß der britische Labour-Vorsitzende Blair keinem Missverständnis auf, als er der Ausbreitung der britischen (mitbestimmungsfreien) Rechtsform der Ltd. über ganz Europa das Wort redete; ${ }_{i}^{\underline{63}}$ die konservativen und christdemokratischen EP-Abgeordneten aus Ländern wie Belgien, Deutschland und Österreich handelten nicht gegen ihre "wahren" Interessen, als sie ihren Nachbarn zur linken der jeweiligen Parteienspektren im Jahr 2001 folgten und die relativ wirtschaftsliberale Fassung der Übernahmerichtlinie zu Fall brachten; ${ }^{64}$ und jene osteuropäischen und britischen Abgeordneten und Regierungen handelten in der Tat im Sinne

Soziale Demokratie? Die politökonomische Heterogenität Europas als Determinante des demokratischen und sozialen Potenzials der EU (EuR-Bei 2013,69)

ihrer Wählerinnen und Wähler, als sie - unbeschadet ihrer Zugehörigkeit zu Parteienfamilien - im Zuge der Verhandlungen über die Dienstleistungsrichtlinie für weitergehende Liberalisierungen der europäischen Dienstleistungsmärkte stritten. ${ }^{\underline{65}}$

Das Fazit ist ernüchternd: Die fehlenden Voraussetzungen eines europäischen Parteiensystems sind nicht nur technischer und nicht nur diskursiver Natur. Die faktische Konfliktstruktur auf dem europäischen Kontinent lässt die Entstehung einer dominanten transnationaleuropäischen Links-rechts-Konfliktachse unwahrscheinlich erscheinen. Hält diese Einschätzung, dann sind der Entwicklung europäischer Parteien, die den europäischen Bürgerinnen und Bürgern rivalisierende politische Programme zur Entscheidung vorlegen und sie im Erfolgsfall in materielle Politik umsetzen könnten, vorerst enge Grenzen gesetzt. $\underline{66}, \underline{67}$

Hieraus folgt eine zweite Implikation: Noch so wünschenswerte Stärkungen des EP im politischen System Europas bewirken nicht zwingend ein Mehr an demokratischer Legitimation europäischer Politik. Noch in seinem Maastricht-Urteil hatte das Bundesverfassungsgericht (BVerfG) der europäischen Politik einen über den Ausbau der Rechte des Europäischen Parlaments hergestellten Zugewinn an Legitimation attestiert. In dem Maße, in dem "sein Einfluss auf die Politik und die Rechtsetzung der Europäischen Gemeinschaft wüchse", $\underline{\text {, }}$ entstünde ein zusätzlicher Kanal der Legitimationszufuhr (die so genannte "duale", also nicht mehr nur über den Umweg der mitgliedsstaatlichen Demokratien bereitgestellte Legitimation). ${ }^{69}$ Die Aufwertungen des EP durch das

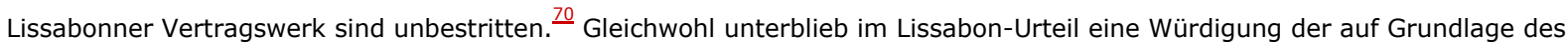


Maastricht-Urteils doch eigentlich zu konstatierenden Legitimitätsfortschritte. Ganz im Gegenteil stellte das BVerfG in einer der umstrittensten ${ }^{71}$ Passagen des Urteils fest: „Der Vertrag von Lissabon führt nicht auf eine neue Entwicklungsstufe

Soziale Demokratie? Die politökonomische Heterogenität Europas als Determinante des demokratischen und sozialen Potenzials der EU (EuR-Bei 2013,69)

der Demokratie." $\underline{72}$ Auch angesichts der in diesem Beitrag geführten Argumentation ist der Zugewinn an Legitimation über das gestärkte EP skeptisch zu beurteilen. Ähnliches gilt beispielsweise für die Rufe nach „mehr Bürgernähe" oder für die Hoffnungen auf den europäischen Bürgerentscheid: Angesichts der politökonomischen Fragmentierung der EU und der damit einhergehenden Konfliktstrukturen bewirken solche Reformen nicht zwingend ein Mehr an Demokratie.

Ist die hier geführte Argumentation also geeignet, die skeptische Position des BVerfG mit zusätzlichen Argumenten zu unterfüttern, so stellt sich doch die Frage, was im europäischen Demokratiedefizit Ursache ist und was Symptom. Im Lissabon-Urteil hatte das BVerfG das Problem der ungleichen Repräsentation (zwischen den Mitgliedsstaaten variierendes Verhältnis aus Wahlberechtigten und gewählten EP-Abgeordneten) in das Zentrum seiner Überlegungen gerückt. Das ungleiche Wahlrecht ist eines der sichtbarsten Anzeichen der "erheblichen Überföderalisierung" $\underline{73}$ des politischen Systems der EU. Diese politische Überföderalisierung aber mag der faktischen politökonomischen Konfliktstruktur Europas gerade angemessen sein. ${ }^{74}$ Würde das Problem der ungleichen Repräsentation beseitigt - wobei unklar ist, wie eine für alle Seiten tragbare Lösung eigentlich aussehen könnte -, blieben alle Hemmnisse der Entstehung kohärenter europäischer Parteien und damit einer demokratischen Steuerung über Wahlen gleichwohl erhalten. Versuche, das demokratische Potenzial der EU durch politisch-institutionelle Reformen zu erweitern, können sich deshalb als etatistische Fehlschlüsse erweisen. $\underline{75}$

Die dritte Implikation ist praktisch-politischer Natur. Wachsen die Potenziale einer europäischen Sozialen Demokratie, wenn es - wie in der Einleitung zu diesem Beitrag anheimgestellt - ihren Verfechtern gelingt, politische Mehrheiten für ihre Ideen zu mobilisieren? Dieser Vorstellung liegt die Prämisse zugrunde, Europa verfüge über ein im Prinzip funktionsfähiges demokratisches System, und zur Verwirklichung der Sozialen Demokratie fehlten lediglich die geeigneten Mehrheitsverhältnisse. Tatsächlich aber würde selbst eine neue Phase sozialdemokratischer Dominanz in EP und Mitgliedstaaten den marktliberalen Drall der europäischen Integration nicht in sein Gegenteil verkehren oder auch nur spürbar abschwächen.

Die letzte Phase sozialdemokratischer Dominanz in Europa fiel auf die Jahre zwischen 1997 und 2003 und damit auf einen Zeitraum vor der EU-Osterweiterung, im Zuge derer die wirtschaftlichen und sozialen Interessengegensätze zwischen den Mitgliedstaaten erheblich zugenommen haben. ${ }^{76}$ Die späten neunziger und frühen

Soziale Demokratie? Die politökonomische Heterogenität Europas als Determinante des demokratischen und sozialen Potenzials der EU (EuR-Bei 2013, 69)

2000er Jahre stellten also ungleich günstigere Randbedingungen einer Korrektur des wirtschaftsliberalen Dralls der europäischen Integration bereit, als sozialdemokratische Regierungen sie heute vorfinden würden. Zu einem Transfer sozialer Regulierung auf die europäische Ebene, einer Verständigung auf Mindeststandards bei der Arbeitnehmermitbestimmung oder etwa einer Einigung auf Mindestsätze bei der Kapitalbesteuerung kam es dennoch nicht. Vielmehr reagierte die europäische Politik mit der Ingangsetzung und Stärkung weicher, rechtlich unverbindlicher Koordinationsformen. ${ }^{77}$ Angesichts der seither zugenommen Interessendivergenzen wären von einer neuen Phase sozialdemokratischer Dominanz weder spürbare Schritte verbindlicher sozialer Integration, noch nachgelagerte politische Korrekturen rechtlicher Ausweitungen der Anwendungsbereiche von Wettbewerbsrecht und Grundfreiheiten zu erwarten. Kurz: Der Mangel an europäischer Sozialer Demokratie ist kein Problem fehlender politischer Mehrheiten. Er ist in die europäische Integration strukturell eingeschrieben.

Dieser skeptischen Einschätzung mag man einen Mangel an Idealismus und Zweckoptimismus vorhalten. Auf absehbare Zeit scheinen mir die Gefahren aus überschießendem Zweckoptimismus folgender Fehleinschätzungen allerdings manifester als der Mangel an politischem Schwung, der sich aus Zweckoptimismus möglicherweise schöpfen ließe. Ungerechtfertigter Zweckoptimismus läuft Gefahr, einer gravierenden Fehleinschätzung Vorschub zu leisten: dem unhinterfragten Glauben, destruktive Eingriffe des europäischen Rechts in die nationalen Bestände an Sozialer Demokratie seien ein Übergangsphänomen und mit Nachsicht zu beurteilen, weil die europäische Soziale Demokratie ohnehin im Entstehen begriffen sei. Der hier angemahnte nüchterne Blick auf die derzeit und absehbar wirksamen Integrationsdynamiken kann hingegen davor bewahren, den Schutz Sozialer Demokratie dort zu vernachlässigen, wo sie sich bis auf weiteres manifestiert: in den sehr heterogenen demokratischen und sozialen Ordnungen der Mitgliedstaaten.

Soziale Demokratie? Die politökonomische Heterogenität Europas als Determinante des demokratischen und sozialen Potenzials der EU (EuR-Bei 2013, 69)

Soziale Demokratie? Die politökonomische Heterogenität Europas als Determinante des demokratischen und sozialen Potenzials der EU (EuR-Bei 2013, 69) 
${ }^{*}$ Apl. Prof. Dr. phil., Politikwissenschaftler und Forschungsgruppenleiter am Kölner Max-Planck-Institut für Gesellschaftsforschung.

${ }^{1}$ In diesem Beitrag führe ich eine Argumentation weiter, die ich in Teilen gemeinsam mit meinem Kollegen Armin Schäfer am Kölner Max-Planck-Institut für Gesellschaftsforschung entwickelt habe. Armin Schäfer stand deshalb auch bei der Abfassung dieses Beitrags Pate. Vergleiche hierzu M. Höpner/A. Schäfer, Grundzüge einer politökonomischen Perspektive auf die europäische Integration, in: M. Höpner/A. Schäfer (Hrsg.), Die Politische Ökonomie der europäischen Integration, 2008, S. $11 \mathrm{ff}$;; M. Höpner/A. Schäfer, A New Phase of European Integration: Organized Capitalisms in Post-Ricardian Europe, West European Politics 33 (2010), S. 344 ff.; M. Höpner/A. Schäfer, Polanyi in Brussels? Embeddedness and the Three Dimensions of European Economic and Social Integration, MPIfG Discussion Paper 2010/8. Für ihre Hilfestellungen danke ich zudem Jürgen Bast, Florian Rödl, Fritz W. Scharpf und Maximilian Waclawczyk.

${ }^{2}$ Vergleiche Kap. 13 in M. G. Schmidt, Demokratietheorien. Eine Einführung. 4. Auflage 2008, S. 225 ff.; T. Meyer, Soziale Demokratie. Eine Einführung, 2009.

${ }^{3}$ Hierzu R. Mayntz, Politische Steuerung: Aufstieg, Niedergang und Transformation einer Theorie, in: K. von Beyme/C. Offe (Hrsg.), Politische Theorien in der Ära der Transformation. PVS-Sonderheft 26, 1996, S. 148 ff.

${ }^{4}$ Ein Versuch der Messung unterschiedlicher Ausmaße an Sozialer Demokratie in entwickelten Industrieländern findet sich in T. Meyer, Praxis der Sozialen Demokratie, 2006, S. 486 ff.

${ }^{5}$ Deutscher Bundestag, 16. Wahlperiode, 157. Sitzung vom 24.4.2008, S. 16452.

${ }^{6}$ Hierzu jüngst K. Busch, Das Korridormodell - relaunched. Ein Konzept zur Koordinierung wohlfahrtsstaatlicher Politiken in der EU, Reihe Internationale Politikanalyse der Friedrich Ebert Stiftung, 2011.

7 Ich verwende die Bezeichnung „judikative Integration" (alternative Bezeichnung: „Integration durch Recht") als deutsche Übertragung des engl. Terminus „integration through law". Der Terminus bezeichnet ein Forschungsprogramm, das jene Integrationswirkungen in das Zentrum des Interesses rückt, die nicht durch politische Übereinkünfte, sondern durch die Interpretation des europäischen Rechts durch Kommission und EuGH erzielt werden. Grundlegend hierzu M. Cappelletti/M. Seccombe/J. Weiler (Hrsg.), Integration Through Law. Europe and the American Federal Experience. Vol. I: Methods, Tools and Institutions, 1986. Einen guten Überblick bietet U. Haltern, Integration durch Recht, in: H.-J. Bieling/M. Lerch (Hrsg.), Theorien der europäischen Integration, 2006, S. 399 ff.

${ }^{8}$ Datenquelle: Eurostat, Europe in Figures. Eurostat Yearbook 2010, 2010, S. 97.

${ }^{9}$ F. Rödl, Arbeitsverfassung, in: A. von Bogdandy/J. Bast (Hrsg.), Europäisches Verfassungsrecht. Theoretische und dogmatische Grundzüge. 2. Auflage 2009, S. 855 (884).

${ }^{10}$ Diese Terminologie stammt von Peter A. Hall und David Soskice, siehe dies., An Introduction to Varieties of Capitalism, in: dies. (Hrsg.), Varieties of Capitalism. Institutional Foundations of Comparative Advantage, 2001, S. $1 \mathrm{ff}$.

${ }^{11}$ Datenquelle: Eurostat (Fn. 8), S. 336.

${ }^{12}$ K. Schubert/S. Hegelich/U. Bazant (Hrsg.), Europäische Wohlfahrtssysteme. Ein Handbuch, 2008.

${ }^{13}$ M. Höpner, Unternehmensmitbestimmung unter Beschuss. Die Mitbestimmungsdebatte im Licht der sozialwissenschaftlichen Forschung, MPIfG Discussion Paper 2004/8, S. 40.

${ }^{14}$ Datenquelle: ETUC, Trade Unions and Industrial Relations in EU Countries, 2010, S. 4.

${ }^{15}$ Die umfassendste Datenquelle hierzu ist J. Visser, ICTWSS Database. Database on Institutional Characteristics of Trade Unions, Wage Setting, State Intervention and Social Pacts in 34 Countries between 1960 and 2007. Version 2, 2009 (http://www.uvaaias.net/208)

${ }^{16}$ Datenquelle: ETUC (Fn. 14), S. 5.

${ }^{17}$ So Hall und Soskice (Fn. 10)

${ }^{18}$ G. Esping-Andersen, The Three Worlds of Welfare Capitalism, 1990.

${ }^{19}$ B. Amable, The Diversity of Modern Capitalism, 2003.

${ }^{20}$ A. Nölke/A. Vliegenthart, Enlarging the Varieties of Capitalism. The Emergence of Dependent Market Economies in East Central Europe, World Politics 62 (2009), S. 670 ff.

${ }^{21}$ J. H. H. Weiler, The Community System. The Dual Character of Supranationalism, Yearbook of European Law 1 (1981), S. 257 ff.; F. W. Scharpf, The Joint-Decision Trap: Lessons from German Federalism and European Integration, Public Administration 66 (1988), S. 239 ff.; F. W. Scharpf, The Joint-Decision Trap Revisited, Journal of Common Market Studies 44 (2006), S. 845.

22 P. Dann, Die politischen Organe, in: A. von Bogdandy/J. Bast (Fn. 9), S. 335 (350 ff.).

${ }^{23}$ Beispielsweise RL 89/391/EWG des Rates vom 12.6.1989.

${ }^{24}$ Beispielsweise RL 2004/35/EG des EP und des Rates vom 21.4.2004.

https://beck-online.beck.de/default.aspx?typ=reference \&y=300\&z=EUR-BEI\&h=1\&b... 07.05 .2014 
${ }^{25}$ Beispielsweise RL 93/13/EWG des Rates vom 5.4.1993.

${ }^{26}$ Siehe ausführlich F. W. Scharpf, Regieren in Europa: Effektiv und demokratisch?, 1999, S. $71 \mathrm{ff}$.

${ }^{27}$ Ebd.

${ }^{28}$ RL 96/34/EG des Rates vom 3.6.1996.

${ }^{29}$ RL 93/104/EG des Rates vom 23.11.1993, novelliert durch RL 2003/88/EG des EP und des Rates vom 4.11.2003.

${ }^{30}$ A. Schäfer, Die neue Unverbindlichkeit. Wirtschaftspolitische Koordinierung in Europa, 2005.

${ }^{31}$ A. Schäfer, Aufstieg und Grenzen der Offenen Methode der Koordinierung, WSI-Mitteilungen 59 (2006), S. 540 ff.; M. Heidenreich/G. Bischoff, Die offene Methode der Koordinierung: Ein europäisches Instrument zur Modernisierung nationaler Sozial- und Beschäftigungsordnungen?, in: M. Heidenreich (Hrsg.), Die Europäisierung sozialer Ungleichheit, 2006, S. 277 ff.

${ }^{32}$ RL 2004/25/EG des EP und des Rates vom 21.4.2004.

${ }^{33}$ RL 2006/123/EG des EP und des Rates vom 12.12.2006.

${ }^{34}$ H. Rasmussen, On Law and Policy in the European Court of Justice. A Comparative Study in Judicial Policymaking, 1986; K. J. Alter, Establishing the Supremacy of European Law, 2001; J. H. Weiler, A Quiet Revolution. The European Court of Justice and its Interlocutors, Comparative Political Studies 26 (2004), S. $510 \mathrm{ff}$.

${ }^{35}$ EuGH, Rs. C-55/94 (Gebhard), Slg. 1995, I- $\underline{4165}$.

${ }^{36}$ Vergleiche für die Auswirkungen von Niederlassungsfreiheit und Unionsbürgerschaft auf das mitgliedsstaatliche Staatsangehörigkeitsrecht jüngst EuGH, Rs. C-135/08 (Rottmann), Slg. 2010, I-1449.

${ }^{37}$ Hinzuzufügen wäre, dass entsprechende Eingriffe auch dort stattfinden, wo transnationale Bezüge der vorgefundenen Beschränkungen allenfalls schwach erkennbar sind. Wird das grenzüberschreitende Erfordernis weit interpretiert, wandeln sich die Grundfreiheiten von Instrumenten der Gewährleistung des grenzüberschreitenden Austauschs von Produktionsfaktoren zu allgemeinen Instrumenten der Deregulierung. Siehe für den Bereich der Warenverkehrsfreiheit M. Büchele, Diskriminierung, Beschränkung und Keck-Mithouard - die Warenverkehrsfreiheit, in: G. Roth/P. Hilpold (Hrsg.), Der EuGH und die Souveränität der Mitgliedstaaten. Eine kritische Analyse richterlicher Rechtsschöpfung auf ausgewählten Rechtsgebieten, 2008, S. 335 (350).

${ }^{38}$ E. Kocher, Das "Soziale" in der Rechtsprechung des Europäischen Gerichtshofs, in: Friedrich Ebert Stiftung (Hrsg.), Der EuGH und das soziale Europa. Für eine Aufwertung sozialer Grundrechte im EU-Rechtssystem, 2009, S. 38.

${ }^{39}$ C. Joerges/F. Rödl, Informal Politics, Formalised Law and the "Social Deficit" of European Integration: Reflections after the Judgments of the ECJ in Viking and Laval, European Law Journal 15 (2009), S. $1 \mathrm{ff}$.

${ }^{40}$ F. Rödl, Wirklich auf einem "guten Mittelweg"?, in: Friedrich Ebert Stiftung (Fn. 38), S. 42 f.

${ }^{41}$ Zu beachten ist zudem, dass die Grundfreiheiten-Judikatur des EuGH auch dann indirekte Wirkungen auf die Balance von Staat und Markt entfaltet, wenn Bereiche außerhalb der Sozialpolitik im engeren Sinne erfasst werden. Als Beispiel sei das untrennbar mit der Mitbestimmungsfrage verknüpfte Gesellschaftsrecht genannt. Ein anderer, besonders sensibler Bereich ist die Steuerpolitik, namentlich die Kapitalbesteuerung, die ebenfalls von der EuGH-Rechtsprechung zu den Grundfreiheiten tangiert wird. Diese Linie der Rechtsprechung entfaltet indirekte Wirkungen auf den Grad an Progression der Einkommensbesteuerung und damit sowohl auf den redistributiven Charakter der mitgliedsstaatlichen Steuersysteme als auch insgesamt auf die Einnahmeseite der europäischen Wohlfahrtsstaaten. Siehe hierzu S. Ganghof/P. Genschel, Deregulierte Steuerpolitik: Körperschaftssteuerwettbewerb und Einkommensbesteuerung in Europa, in: M. Höpner/A. Schäfer (Fn. 1), S. 311 ff.

42 EuGH, Rs. C-271/08 (Kommission/Deutschland), Slg. 2010, I- $\underline{7091}$.

${ }^{43}$ Einen guten Überblick bietet A. Dyevre, Unifying the Field of Comparative Judicial Politics: Towards a General Theory of Judicial Behaviour, European Political Science Review 2 (2010), S. 297 ff.

${ }^{44}$ T. L. Brunell/A. Stone Sweet, Overriding the European Court of Justice? Qualitative Evidence from Cases in the Carruba, Gabel, and Hankla Dataset (1987-1997), Yale Law School Public Law Working Paper 217, 2010.

${ }^{45}$ Scharpf (Fn. 26), S. $71 \mathrm{ff}$.

${ }^{46}$ G. Rosenberg, Judicial Independence and the Reality of Political Power, The Review of Politics 54 (1992), S. 369 ff.; T. S. Clark, The Separation of Powers, Court Curbing, and Judicial Legitimacy, American Journal of Political Science 53 (2009), S. $971 \mathrm{ff}$.

${ }^{47}$ Vergleiche ausführlich M. Höpner/A. Schäfer, Grenzen der Integration - wie die Intensivierung der Wirtschaftsintegration zur Gefahr für die politische Integration wird, Integration 33 (2010), S. 3 (15 ff.).

${ }^{48}$ Als Beispiele seien die EuGH-Entscheidungen Rs. C-144/04 (Mangold), Slg. 2005, I-9981, Rs. C-267/06 (Maruko), Slg. 2008, I1757, und Rs. C-303/06 (Coleman), Slg. 2008, I- $\underline{5603}$, angeführt.

${ }^{49}$ F. W. Scharpf, Individualrechte gegen nationale Solidarität, in: M. Höpner/A. Schäfer (Fn. 1), S. 89 (94).

${ }^{50}$ Ein Durchbruch war in dieser Hinsicht die Entscheidung im Fall EuGH, Rs. C-184/99 (Grzelczyk), Slg. 2001, I- $\underline{6193}$

51

https://beck-online.beck.de/default.aspx?typ=reference \&y=300\&z=EUR-BEI\&h=1\&b... 07.05.2014 
Vergleiche beispielsweise F. C. Mayer, Der EuGH als Feind? Die Debatte um das soziale Europa in der europäischen Rechtsprechung, Integration 32 (2009), S. 246 (250 f.) mit Scharpf, (Fn. 45), 91 ff., sowie A. J. Menéndez, European Citizenship after Martinez Sala and Baumbast. Has European Law Become More Human but Less Social?, ARENA Working Paper 2009/11.

52 Alle Zahlenangaben in diesem Abschnitt basieren auf den Daten des Sachverständigenrats zur Begutachtung der gesamtwirtschaftlichen Entwicklung (http://www.sachverstaendigenrat-wirtschaft.de/statistik.html).

${ }^{53}$ H. Enderlein, Nationale Wirtschaftspolitik in der europäischen Währungsunion, 2004, S. 66 ff.

${ }^{54}$ D. Soskice, Wage Determination: The Changing Role of Institutions in Advanced Industrialized Countries, Oxford Review of Economic Policy 6 (1990), S. 36 ff.; P. A. Hall/R. J. Franzese, Mixed Signals: Central Bank Independence, Coordinated Wage Bargaining, and European Monetary Union, International Organization 52 (1998), S. 505 ff.; L. Kenworthy, Corporatism and Unemployment in the $1980 \mathrm{~s}$ and 1990 s, American Sociological Review 67 (2002), S. $367 \mathrm{ff}$.

${ }^{55}$ Siehe zu diesen Versuchen H. Enderlein, Adjusting to EMU. The Impact of Supranational Monetary Policy on Domestic Fiscal and Wage-Setting Institutions, European Union Politics 7 (2006), S. 113 ff.; A. Hassel, Wage Setting, Social Pacts and the Euro. A New Role for the State, 2006.

${ }^{56}$ Zum Zeitpunkt der abschließenden Bearbeitung des vorliegenden Beitrags (im September 2011) ist die Ausgestaltung der Instrumente zur makroökonomischen Überwachung im Euro-Raum noch offen. Vergleiche hierzu den Vorschlag für eine Verordnung über die wirksame Durchsetzung der haushaltspolitischen Überwachung im Euroraum (KOM (2010) $\underline{525}$ endg.) und den Vorschlag für eine Verordnung über Durchsetzungsmaßnahmen zur Korrektur übermäßiger makroökonomischer Ungleichgewichte im Euroraum (KOM (2010) 527 endg.).

${ }^{57}$ Ungefähr zwei Drittel des Wachstums der Staatsquoten westlicher Industrieländer seit 1950 beruht auf der Expansion der Wohlfahrtsstaaten. Siehe M. G. Schmidt/F. Wolf, Expansion und Reform der sozialen Sicherungssysteme 1945-2005, in: M. G. Schmidt et al. (Hrsg.), Der Wohlfahrtsstaat. Eine Einführung in den historischen und internationalen Vergleich, 2007, S. $240 \mathrm{ff}$.

${ }^{58}$ Vergleiche hierzu M. Höpner/A. Schäfer, Polanyi in Brussels? (Fn. 1).

${ }^{59}$ Siehe etwa K. Eder, Öffentlichkeit und Demokratie, in: M. Jachtenfuchs/B. Kohler-Koch (Hrsg.), Europäische Integration. 2. Auflage 2003, S. 85 (116); D. Grimm, Braucht Europa eine Verfassung?, Juristen-Zeitung 50 (1995), S. 581 , (585 ff.); P. G. Kielmansegg, Integration und Demokratie (mit Nachwort zur 2. Auflage), in: M. Jachtenfuchs/B. Kohler-Koch (ebd.), S. 49 (57 ff.).

${ }^{60}$ Widerspruch findet sich aber bei Zuleeg: „Empirische Belege für mangelhafte Randbedingungen der Demokratie sind nicht erbracht." M. Zuleeg, Die Vorzüge der Europäischen Verfassung, in: A. von Bogdandy/J. Bast (Fn. 9), S. 1045 (1057).

${ }^{61}$ Im Sinne einer grundsätzlichen Überwindbarkeit solcher Barrieren durch verstärkte transnationale Kommunikationsanstrengungen argumentieren: J. Habermas, Ach, Europa, 2008, S. 106 ff.; S. Oeter, Föderalismus und Demokratie, in: A. von Bogdandy/J. Bast (Fn. 9), S. 73 (98 f.).

62 Dieses Problem greift vor allem dort, wo europäische Politiken in die Grundprinzipien der mitgliedstaatlichen Produktions- und Verteilungsregime einzugreifen drohen.

${ }^{63}$ Börsen-Zeitung vom 15. September 2004, S. 2.

${ }^{64}$ Siehe ausführlich: H. Callaghan/M. Höpner, European Integration and the Clash of Capitalisms. Political Cleavages over Takeover Liberalization, Comparative European Politics 3 (2005), S. 307 (323).

${ }^{65}$ M. Höpner/A. Schäfer, Grenzen der Integration - wie die Intensivierung der Wirtschaftsintegration zur Gefahr für die politische Integration wird, Integration 33 (2010), S. 3 (19); S. Hix/A. Noury, After Enlargement: Voting Patterns in the Sixth European Paliament, Legislative Studies Quarterly 34 (2009), S. 159 (169 ff.).

${ }^{66}$ Vergleiche etwa Erhard Epplers Plädoyer für eine Europäisierung der Sozialdemokratie. E. Eppler, Epochenwende. Über die Notwendigkeit des Aufbaus einer sozialen Demokratie in Europa, Reihe Internationale Politikanalyse der Friedrich Ebert Stiftung, 2010, S. 10.

${ }^{67}$ Der Standardeinwand, dass ein funktionsfähiges politisches System ja nicht dem Idealtyp der Mehrheitsdemokratie (mit regelmäßiger Alternanz zwischen vergleichsweise säuberlich getrennter Regierung und Opposition) entsprechen müsse, ginge hier ins Leere. Auch die Verhandlungsdemokratie (Konsensusdemokratie, Konkordanzdemokratie) ist, um Demokratieerfordernissen zu genügen, zunächst einmal darauf angewiesen, dass sich die dominanten Konfliktlinien entlang der Programme sortieren, die den Wählerinnen und Wählern bei der Wahlentscheidung vorgelegt werden. In gleich welchem politischen System fungiert also eine Mindestkongruenz von Parteien und faktischer Konfliktstruktur als Demokratievoraussetzung. Grundlegend zur Unterscheidung von Mehrheits- und Verhandlungsdemokratien: A. Lijphart, Patterns of Democracy. Government Forms and Performance in Thirty-Six Countries, 1999.

${ }^{68}$ BVerfGE 89, $\underline{155}, \underline{185}$.

${ }^{69}$ A. von Bogdandy, Grundprinzipien, in: A. von Bogdandy/J. Bast (Fn. 9), S. 13 (62 ff.).

${ }^{70}$ S. Oeter (Fn. 61), S. 92.

${ }^{71}$ Vergleiche für die heftige Kritik an diesem Teil der Lissabon-Entscheidung beispielhaft M. Selmayr, Endstation Lissabon? Zehn Thesen zum „Niemals"-Urteil des Bundesverfassungsgerichts vom 30. Juni 2009, ZEuS 12 (2009), S. 637 (647 ff.).

https://beck-online.beck.de/default.aspx?typ=reference \&y=300\&z=EUR-BEI\&h=1\&b... 07.05 .2014 
72 BVerfGE 123, 267, S. 379 ff. Vergleiche hierzu beispielsweise Schoo: „Die Europäische Union erfährt durch den Vertrag von Lissabon eine deutliche Verbesserung ihrer demokratischen Legitimation". J. Schoo, Das neue institutionelle Gefüge der EU, in: J. Schwarze/A. Hatje (Hrsg.), Der Reformvertrag von Lissabon. EuR Beiheft 1/2009, S. 51 (68).

${ }^{73}$ Ebd., Rn. 288.

${ }^{74}$ Im Ergebnis ist - wenn auch mit abweichender Stoßrichtung - Stefan Oeter zuzustimmen: "Vieles spricht dafür, dass die Europäische Union genau die Verfassung hat, die sie verdient." S. Oeter (Fn. 61), S. 118. Siehe auch F. Rödl, in diesem Heft.

75 Vergleiche Grimms Warnung vor "etatistischen Verkürzungen" in der Debatte über das europäische Demokratiedefizit: D. Grimm (Fn. 59), S. 587.

${ }^{76}$ Siehe hierzu P. Manow/A. Schäfer/H. Zorn, Europe's Party-political Centre of Gravity, 1957-2003, Journal of European Public Policy 15 (2008), S. $20 \mathrm{ff}$.

77 A. Schäfer (Fn. 31); ders., A New Form of Governance? Comparing the Open Method of Coordination to Multilateral Surveillance by the IMF and the OECD, Journal of European Public Policy 13 (2006), S. $70 \mathrm{ff}$. 